\title{
Plant survival monitoring with UAVs and multispectral data in difficult access afforested areas
}

\begin{abstract}
Water supply devices enable afforestation in dry climates and on poor lands with generally high success rates. Previous survival analyses have been based on the direct observation of each individual plant in the field, which entails considerable effort and costs. This study provides a low-cost method to discriminate between live and dead plants in afforestations that can efficiently replace traditional field inspections through the use of UAVs equipped with RGB and NIR sensors. The method combines the use of a conventional camera with an identical camera modified to record the NIR channel. Survival analysis was performed with digital image processing techniques based on calculated indices associated with plant vigour and PCA-based decorrelation. The method yielded results with high global accuracy rates $(\sim 96.2 \%)$ with a minimum percentage of doubtful plants, even in young plantations (seedlings $<30 \mathrm{~cm}$ tall). The procedure could be particularly useful in hazardous areas.
\end{abstract}

Keywords: UAV, plant control, degraded region, gap detection, Waterboxx 
Afforestation is one of the most studied and widely used methods for combating the effects of climate change (García-Valdés et al. 2015). However, the afforestation of arid or degraded areas faces many challenges, such as the exposure of new plantations to water- and temperature-related stresses. Extreme temperatures, low relative humidity, high evaporation and strong winds are common in degraded areas. Plants must recover from the potential stress experienced during handling and establish root contact with the soil to resume vital functions of water and nutrient absorption in the new environment (Haase \& Rose 1993). Irrigation systems and/or alternative systems (plant protectors, hydrogels, pads, etc.) that provide water to the new plants and protect them from extreme temperature are commonly used to increase plant survival rates during early growth stages. The comparison of irrigation systems from a cost-efficiency perspective has been widely studied in works such as those of Bainbridge, 2002 and Tang, Folmer and Xue, 2015. However, irrigation is almost always economically unviable in forest restoration projects.

The Waterboxx system (Groasis, Netherlands), consists of a container that serves as a water reservoir and provides housing for the seedlings inside (Fig. 1). The water stored in the tank provides thermal protection to the plant and acts as a buffer against high daytime temperatures and possible night frosts. This system is designed to collect rainwater and condensation from nocturnal dew, providing a constant moisture supply to the plant (Marcos-Robles et al. 2013). This type of device offers a viable approach to adapting forest crops to the conditions in degraded lands to improve plantation 
success. The Waterboxx system (www.groasis.com) is supported by experimental results in different regions (Del Campo et al. 2013; Liu et al. 2014). Climate change has made such devices receive increasing attention and interest for afforestation in dry climates or poor lands.

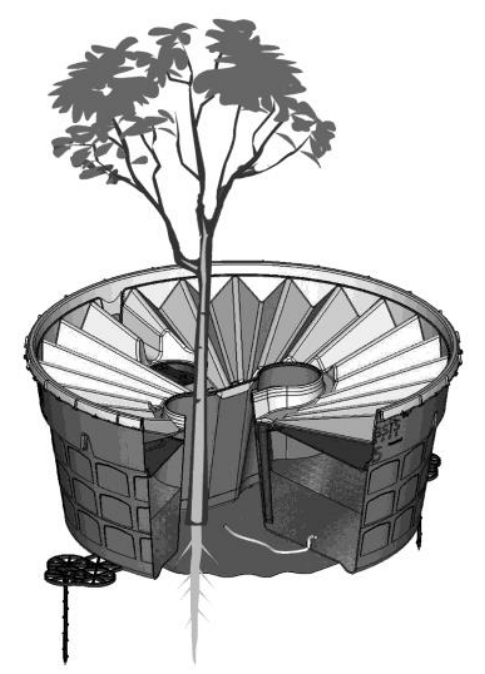

Fig. 1. Waterboxx device. Courtesy of Groasis.

Plant survival analysis is traditionally performed through systematic sampling (Johnson et al. 2008). This method is based on field observation, in which approximately $5 \%$ of the plants are inspected, starting from a randomly selected point and following a certain pattern. The phenological status of each plant is recorded individually, and each sample is georeferenced with a GPS to create a locational inventory of all monitored plants. Annual monitoring should be planned for the first few years. The main problem with the traditional method, apart from the laboriousness entailed, is that checking only a sample of plants does not provide knowledge of the total number of plants that must be replenished. Systematic field sampling is expensive and complex in areas of complicated topography, those that are difficult to access and those with extreme environmental conditions. 
The use of unmanned aerial vehicles (UAVs) is especially popular for forest monitoring and management (Perea-Moreno et al. 2016). These aerial platforms allow sensors or data capture devices to be elevated to a predetermined altitude (Eisenbeiß 2009). The spatial resolution of images (i.e., ground sample distance, GSD) is a function of mainly the height of the flight platform above the ground and the focal length of the camera. An adequate combination of both parameters can provide very high spatial resolution data that enable identification of even small trees and seedlings. Studies such as Mesas-Carrascosa et al. 2015 focus on the establishment of an optimal balance between spatial resolution and spectral discrimination as a requirement to optimize the mission planning and image processing to achieve every plant analysis objective.

In addition to the flight platform itself, the type of sensor used is key in monitoring studies. Vegetation has a unique spectral signature that enables it to be readily distinguished from other types of coverage in multispectral images. In particular, NIR information can be very helpful for discriminating smaller plants from the background due to the high reflectance of vegetation in this spectral region. Multispectral image processing has been widely used in vegetation analysis and classification through the use of indices (Agapiou et al. 2012). The Normalized Difference Vegetation Index (NDVI) (Tucker 1979) is the most widely used index in multispectral remote sensing research for detecting and quantifying vegetation (Lloret et al. 2007; Cristiano et al. 2014; Helman et al. 2015). The Soil-Adjusted Vegetation Index (SAVI) was proposed by Huete (1988) to minimize the effects of soil background on the quantification of greenness by incorporating a soil adjustment factor into the basic NDVI formula. The SAVI and its variants, such as L-SAVI, are typically used in arid zones (Zhou 2014), decreasing the NDVI variability in which the same value of NDVI could correspond to vigorous but sparse cover or to dense but low-vitality cover. All these 
indices are based on the red (R) and near-infrared (NIR) spectral domains. By contrast, indices using the green $(\mathrm{G})$ band are indicators of active chlorophyll production, which is related to leaf nitrogen concentration (Salamí et al. 2014). The Green Normalized Difference Vegetation Index (G-NDVI) is probably the most widely used within this category and is less influenced by factors other than chlorophyll (Hunt et al. 2005). Compared with the NDVI, the G-NDVI is sensitive to plant pigment concentration and can be discriminatory in young plants or plants with little vegetative development, such as those in the present study. The maximum sensitivity of the G-NDVI was demonstrated to occur from $520 \mathrm{~nm}$ to $630 \mathrm{~nm}$ (Gitelson \& Merzlyak 1997).

Studies such as that by Gnädinger and Schmidhalter (2017) confirm the validity of the simplest digital techniques, including contrast enhancement for counting crops, obtaining a close correlation between in situ and image-based plant counts. In addition, spatial contrasts can be softened or reinforced by filtering techniques. Since high frequencies are associated with sharp changes in density, high-pass filters improve edge detection by reinforcing contrast (Chuvieco et al. 2002). The use of the Sobel filter (Sobel \& Feldman 1973), focused on pixel-to-pixel intensity differences, can aid in the detection of contours. Huete and Jackson (1987), Singh and Singh (2011), and Li and Shao (2014) suggest that principal component analysis (PCA) transformation can significantly improve vegetation signal extraction compared with various vegetation indices. One of the most effective enhancements in aerial images with high correlation between bands is the PCA-based "decorrelation stretch" (Gillespie et al. 1986).

To replace field inspections and to increase the efficiency of afforestations, we present a method to identify live plants in Waterboxx plantations from images captured by airborne sensors and image processing techniques. This method is especially useful in areas that are difficult to access, enabling the detection and georeferencing of possible gaps to replace and fill them. 


\section{$2 \quad$ Materials and methods}

\subsection{Study site description}

The monitoring study was performed in approximately 0.50 ha of the total 13.70 ha reforested in the Ski Resort of San Isidro $\left(43^{\circ} 2^{\prime} \mathrm{N}, 5^{\circ} 23^{\prime} \mathrm{W}\right.$, altitude 1,756 MAMSL) within the Provincial Council of León (Spain). The area has a steep slope and an unstable sandy soil with abundant loose rocks. Following the landscape criteria, the plot has been planted with various species of both conifers and hardwoods in Waterboxx devices: Pinus sylvestris (Scots pine), Betula pendula (birch) and Sorbus aucuparia (rowan). In some cases, two species were planted in the same Waterboxx device (Fig. 2a). During pre-field inspection, some dead plants were observed (Fig. 2b), and the average plant height at the time of the flight never exceeded $60 \mathrm{~cm}$.

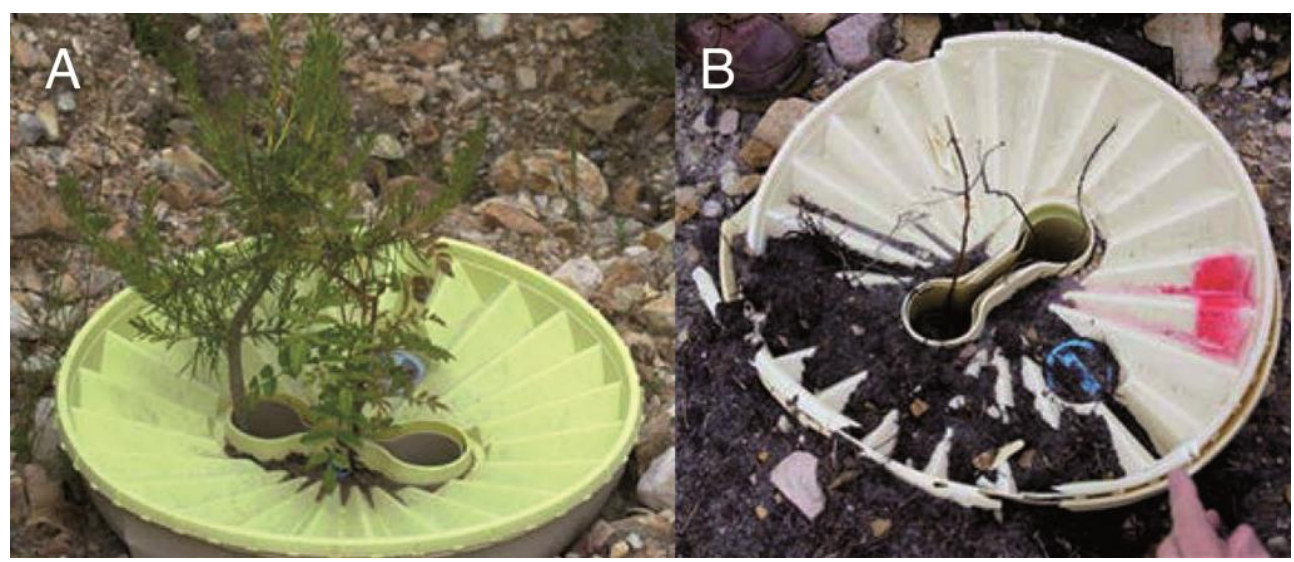

Fig. 2. Waterboxx device with a) two seedlings (pine and rowan) and b) a dead plant (gap). 


\subsection{Flight campaign}

The flight campaign was conducted in the summer of 2015 using a vertical take-off and landing (VTOL) rotating-wing UAV with 8 rotors. The UFOCAMXXL V3 MikroKopter (www.mikrokopter.de) (Fig. 3a) with $2.5 \mathrm{~kg}$ of payload was used. For this experiment, the UAV was equipped with a specially designed gimbal that supported two cameras taking synchronized shots (Fig. 3b): two conventional Olympus (www.olympus-global.com) PEN E-P1 mirrorless cameras with 12.3 megapixels $(4,032 \times 3,024)$ and Live MOS sensor. One of the cameras was modified with a high-pass filter that cut at $720 \mathrm{~nm}$ instead of the original camera's low pass filter. The new filter blocked the visible spectrum recorded by the CCD and enabled the capture of the NIR channel (Lehmann et al. 2015). Both cameras employed M. Zuiko Digital ED 12 mm f/2 lenses. As its sensor is an Olympus micro $4 / 3$ sensor, the lens is equivalent to approximately $24 \mathrm{~mm}$ in a full-format camera. The total cost of this dual-camera system does not exceed $\$ 1,000$ (including modification of the camera and adaptation of the gimbal). However, the market offers fully integrated multispectral systems that are increasingly affordable. The ground control station and the UAV were radio linked, transmitting altitude, position and status data, although the UAV is controlled by an autopilot for fully autonomous flights. The MikroKopter MK Tool control software allowed precise flight path planning with waypoints for the optimal definition of the passes. 


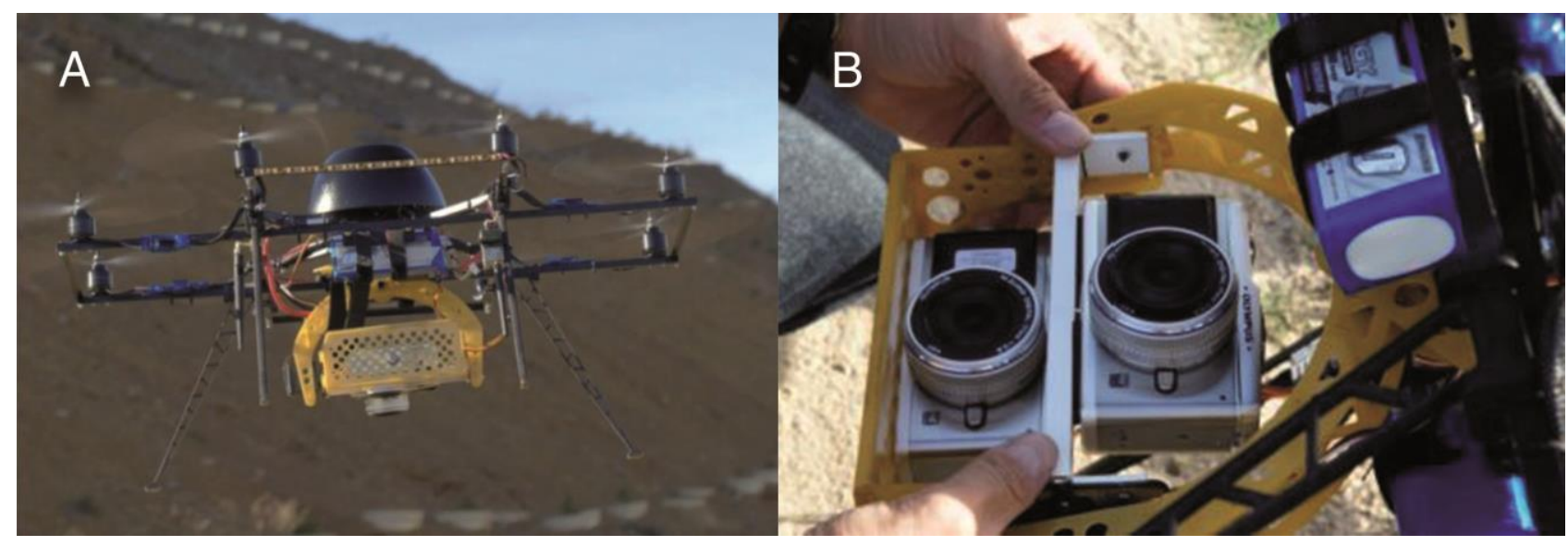

Fig. 3. a) Octocopter UFOCAMXXL8 V3. b) Special gimbal adapter for the two Olympus E-P1 cameras (RGB and modified for NIR) and c) camera positions, image overlap and GCPs distribution.

The flight was performed at an altitude of $109 \mathrm{~m}$, and 201 high-resolution images of the plot (Fig. 3c) with each of the cameras and with overlaps ( $40 \%$ across the flight direction and $70 \%$ along the flight direction) were obtained. A Leica SR530 RTK GPS (3 mm +1 ppm in static mode) was used to take 11 ground control points (GCPs) of predefined identifiable points in the photographs for subsequent geometric correction and georeferencing of the plot mosaics. Although several studies have thoroughly discussed this issue (Tonkin \& Midgley, 2016 and Agüera-Vega, Carvajal-Ramírez and Martínez-Carricondo 2016), there is no distinct, accepted criterion for establishing the number of GCPs. The choice depends largely on the size of the area, among other factors. The results obtained by Agüera-Vega et al. 2016 in a 17.64 ha test field suggest that DSM and orthoimage generation with 15 GCPs yielded optimal horizontal and vertical accuracy. Accordingly, we have tried to use an equivalent GCP density (approx. $0.8 \mathrm{GCPs} / \mathrm{ha}$ ). 


\subsection{Orthoimage generation}

The flight images from each camera were used to generate the corresponding orthomosaics (RGB and NIR) using a PC with a $2.80 \mathrm{GHz}$ Intel Core i7-7700HQ, 32 GB RAM, NVIDIA GeForce GTX 10708 GB and running PhotoScan Professional Edition version 1.4.1. (Agisoft LLC, Russia). This automated image correlation (AIC) software performed the processes of (1) automatic aerial triangulation, (2) bundle block adjustment and (3) orthomosaic creation. The structure from motion (SfM) computation enabled automatic image alignment, calibration and reconstruction of threedimensional scene geometry and camera motion parameters. The program detected image feature points (i.e., geometrical similarities such as object edges or other specific details). The three phases of the process were automated, only requiring the introduction of GCPs at the end of phase (1). A total of 11 GPS points identifiable in several photographs were required to ensure the accuracy of the results and the correct fit of spectral bands between the two shots. All steps of the workflow were set at "very high" input to ensure the maximum resolution possible. The Waterboxx devices selected for method validation were located in the orthomosaic and labelled in a vector layer for identification in the following steps.

\subsection{Image post-processing}

To work with the data from both cameras, the resulting orthoimages were integrated with Geomatica software (PCI-Geomatics, Canada) to obtain a single file with the blue (B), G, R and NIR bands. The use of precise GCPs in the orthoimage generation process ensured an accurate overlap between the visible and NIR channels in the resulting multispectral image. With these data, digital treatments were performed to improve certain image characteristics that enabled the discrimination of live and 
dead plants. Following previous studies (Hunt et al. 2005; Zhou 2014; Helman et al. 2015), the vegetation indices NDVI, G-NDVI and SAVI were tested.

When image channels are highly correlated, conventional enhancements (i.e. independent image contrast modifications) are not an effective approach. A general estimation of band correspondence can be obtained by simple correlation analysis (correlation matrices or scattergrams). On this basis, a contrast enhancement was also performed on the multispectral image via the decorrelation procedure. This 3-dimensional procedure used the full range of colours for the display of an image with less redundancy and maintained most of the information of the original image. However, when the number of image channels $\mathrm{N}$ is greater than three, the projection of the $\mathrm{N}$-dimensional data space onto the three-dimensional colour space requires selection of a part of the available information to reduce the degree of freedom to 3 . The first three components of the PC transformation contain the major possible proportion of the variance in the original image. Therefore, the N-dimensional solution $(\mathrm{N}>3)$ involves PCA. The first three PC channels are linearly transformed, with the threedimensional decorrelation balancing their signal-to-noise ratio (SNR's) while maintaining their decorrelations.

Edge-enhancement filters (Sobel-type filters) were tested on the images to verify their usefulness in the gap detection process, and in the contour identification of the Waterboxx cover and its central aperture. The results obtained by digital treatment of the images were checked in the field to validate the proposed method.

\subsection{Survival analysis}

The actual data of the phenological status of each plant were collected from a sample of 132 devices using visual field inspection. The status of each device was registered and georeferenced with GPS 
to create an inventory of all monitored plants. Results are validated comparing image interpretation with ground-truth observation to compute categorical verification scores (true/false positives and true/false negatives) and the probability of detection, probability of false detection and false alarm ratio metrics.

\section{$3 \quad$ Results and discussion}

\subsection{Flight campaign and orthoimage generation}

The photogrammetric processing of aerial images results in a single fused orthomosaic (RGB and NIR bands) covering the study area with a GSD $\sim 2.74 \mathrm{~cm}_{\text {pixel }}{ }^{-1}$. The overall RMSE value based on GCP $(3.523 \mathrm{~cm})$ after the bundle block adjustment, can be considered acceptable for obtaining a georeferenced positioning of the water supply devices.

The lighting conditions during the flight allowed RGB (Fig. 4a) and NIR (Fig. 4b) orthoimages with good radiometric quality to be obtained. The overlap between these images was somewhat worse than expected due to a slight inclination of the UAV caused by the wind, with a deviation of approximately 2 pixels $(<4 \mathrm{~cm})$. From the total modelled area of 13.70 ha, a pilot area of 0.50 ha was selected for analysis where a total of 132 Waterboxx devices were located. 

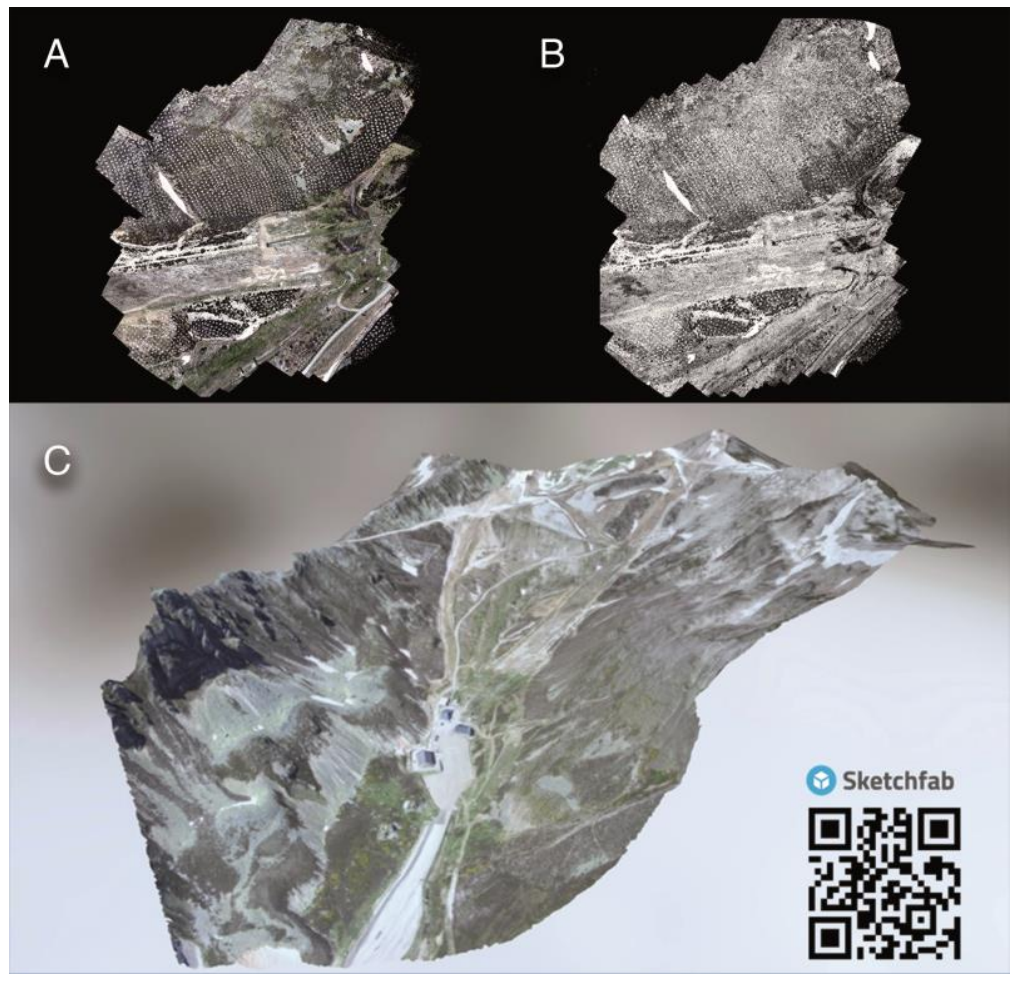

Fig. 4. a) RGB and b) NIR orthomosaics; c) 3D model available at https://skfb.ly/EMqH.

\subsection{Image post-processing and survival analysis}

A composite image with NIR, $\mathrm{R}$ and G channels was created, and the NDVI, G-NDVI and SAVI were determined, which greatly improve detection compared to that achieved with conventional enhancements based on independent contrast modifications or individual input band "stretches". In radiometric terms, some redundancy was identified in the image information with a multivariate statistical analysis (Table 1). The correlation matrix between the four image channels showed a high correlation between the channels of the visible spectrum $(R, G$ and $B)$. This fact usually produces rather dull colour composites because it is not possible to use the full range of colours in the representation. To eliminate this problem and to reduce the dimensionality of the image at the same 
time, a common solution involves PCA transformation. Principal components are not correlated, and therefore radiometric differences between spectral responses are enhanced.

Table 1. Correlation matrix of multispectral images.

\begin{tabular}{ccccc}
\hline & Blue & Green & Red & NIR \\
\hline Blue & 1.0000 & & & \\
Green & 0.9930 & 1.0000 & & \\
Red & 0.9866 & 0.9965 & 1.0000 & \\
NIR & 0.7329 & 0.7597 & 0.7643 & 1.0000 \\
\hline
\end{tabular}
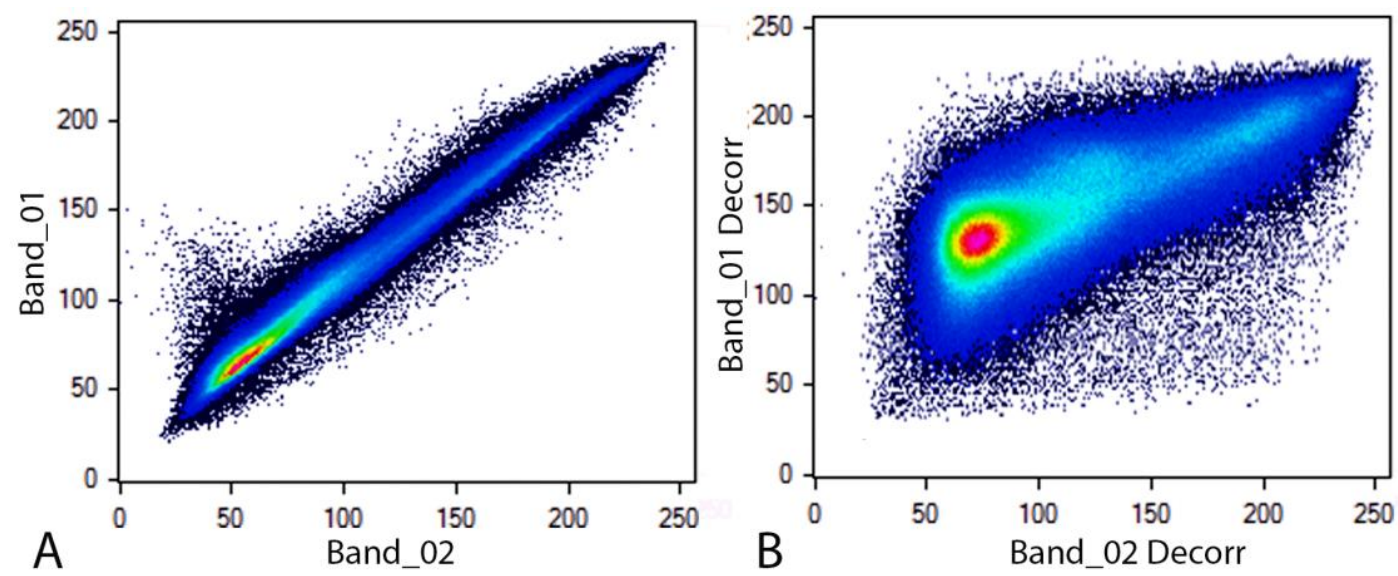

Fig. 5. Comparison between image bands 1 and 2: a) original image scattergram and b) destretched image scattergram. 
Figure 6 shows a comparative example of the digital treatments used on a Waterboxx device in one of the most unfavourable cases. The images show how digital enhancements and selected indices allowed the detection of spectral plant responses that would not have been possible to identify with a real colour image.

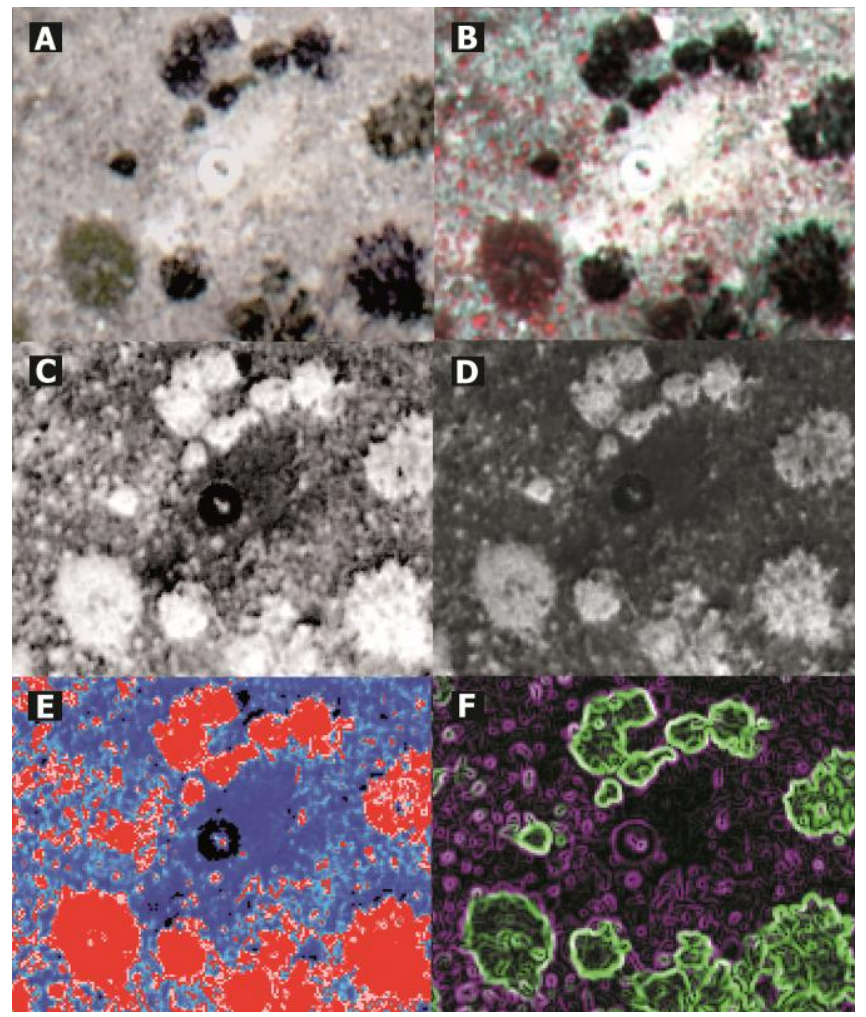

Fig. 6. Digital treatments of a doubtful Waterboxx device: a) visible RGB image; b) false-colour NIR image; c) SAVI image; d) G-NDVI image; e) PCA-based decorrelation enhancement of the image; and f) filter composite (Sobel/Average/Sobel) over NDVI.

Live plants could be identified in the infrared composition (Fig. 6b) in the majority of doubtful cases in the real colour image (Fig. 6a). The vegetation indices (Fig. 6c and 6d) together with the PCAbased decorrelation enhancement (Fig. 6e) offered the best results. The identification of less 
developed seedlings (10-15 cm tall) with indices associated with the level of vigour (NDVI, G-NDVI and SAVI) was more problematic and could cause confusion with dead plants. The differences in interpretation among the plant indices tested were not significant. PCA-based transformation provided better results for small plants, offering a clear signal for live plants (Fig. 6e).

The Waterboxx device hindered the identification of plants with little vigour, generating a similar spectral response in the central aperture. However, for plants with more foliage (e.g., Betula pendula) and larger plants, the uniform reflectivity of the polypropylene flap contrasted with the green colour of the leaves and aided identification. The application of Sobel filters to vegetation indices (Fig. $6 \mathrm{f}$ and 7f) was useful to discriminate between the plant and the central edge of the Waterboxx device. However, to improve the effectiveness of this filter, GSD values of less than $2 \mathrm{~cm}$ would be required to unambiguously identify the water pickup hole of the device. 


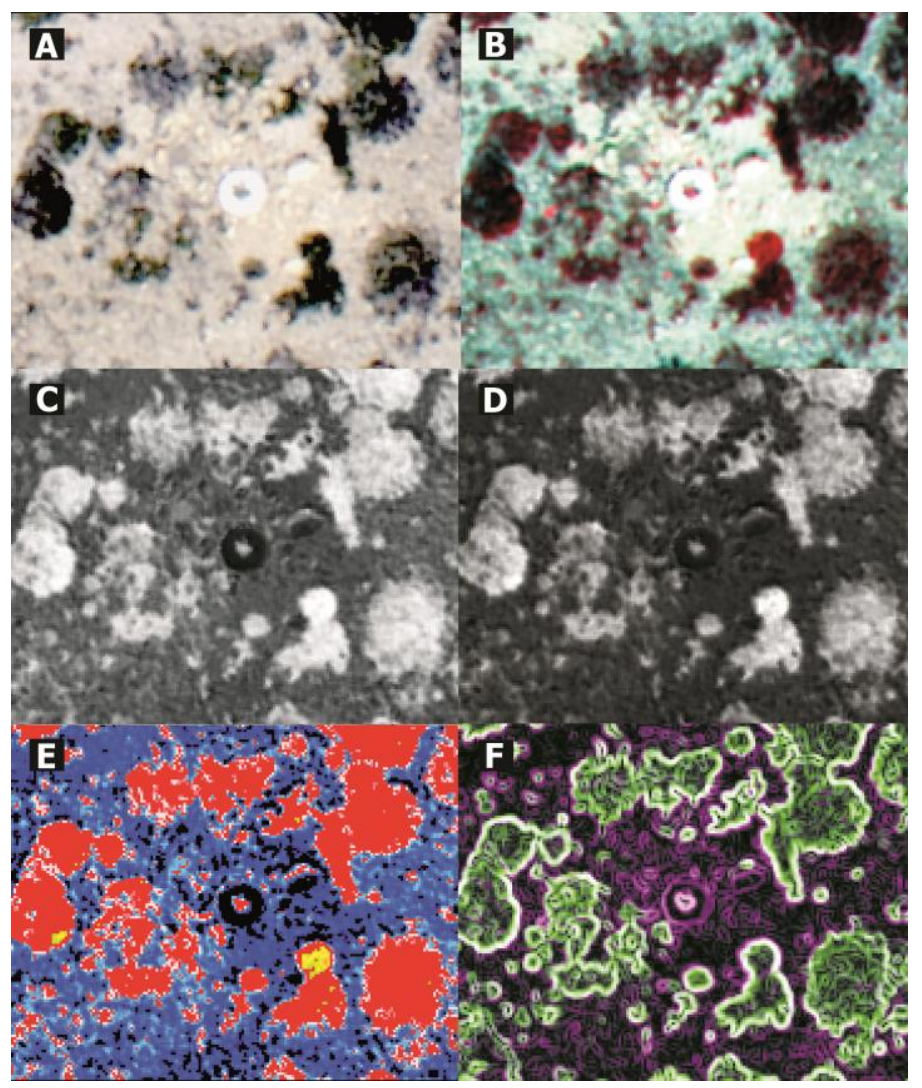

Fig. 7. Digital treatments of a clear positive Waterboxx device: a) visible RGB image; b) falsecolour NIR image; c) SAVI image; d) G-NDVI image; e) PCA-based decorrelation enhancement of the image; and f) filter composite (Sobel/Average/Sobel) over NDVI.

Overall, the image analysis allowed the identification of 126 living plants with 6 doubtful cases out of 132 Waterboxx devices assessed. The doubtful cases were assumed to be gaps because the spectral response was too weak to confirm their survival. The comparison with field inspections of the analysed samples showed that 4 doubtful seedlings were gaps (successes), 2 doubtful seedlings were alive plants partly covered by vegetation that did not allow the correct identification of the plant in the device, and 3 plants considered to be alive were actually false positives (failures). The percentage of doubtful plants was 4.5\% (6/132), and the global accuracy index (total number of hits/total number 
of plants analysed) was $96.2 \%(127 / 132)$. Categorical verification scores were calculated from the confusion matrix (Table 2) showing agreement and disagreement between image-based prediction and observed plant status. The probability of detection (POD) is the percentage of alive plants that were correctly identified as alive using processed images. The probability of false detection (POFD) is the proportion of dead seedlings that were falsely classified as alive. FAR is the proportion of incorrectly predicted alive plants from all devices that were predicted to be alive.

Table 2. Confusion matrix and cross-tabulation-based validation metrics.

\begin{tabular}{|c|c|c|c|}
\hline \multirow{4}{*}{$\begin{array}{l}\text { Image-based } \\
\text { observation }\end{array}$} & \multirow[b]{3}{*}{ Alive } & \multicolumn{2}{|c|}{ Ground truth observation } \\
\hline & & Alive & Gap (confirmed) \\
\hline & & $\begin{array}{l}\text { True Positive (TP) } \\
123 / 132\end{array}$ & $\begin{array}{ll}\text { False Positive (FP) } \\
3 / 132\end{array}$ \\
\hline & Gap (or doubtful) & $\begin{array}{l}\text { False Negative }(\mathrm{FN}) \\
2 / 132\end{array}$ & $\begin{array}{l}\text { True Negative (TN) } \\
4 / 132\end{array}$ \\
\hline Metric & Formula & Computed value & Optimal value \\
\hline $\begin{array}{l}\text { Probability } \\
\text { detection }\end{array}$ & $\mathrm{POD}=\mathrm{TP} /(\mathrm{TP}+\mathrm{FN})$ & 0,984 & 1 \\
\hline $\begin{array}{l}\text { Probability of false } \\
\text { detection }\end{array}$ & $\mathrm{POFD}=\mathrm{FP} /(\mathrm{FP}+\mathrm{TN})$ & 0,429 & 0 \\
\hline False alarm ratio & $\mathrm{FAR}=\mathrm{FP} /(\mathrm{TP}+\mathrm{FP})$ & 0,024 & 0 \\
\hline
\end{tabular}

In doubtful cases, and with less vigorous species or individuals less than $30 \mathrm{~cm}$ in height, an increase in global accuracy would require lowering the flight altitude to increase precision in the analysis. However, when doubtful plants represent a small percentage of the total, reducing the flight altitude would be unnecessary due to its negative impact on the performance of the method. 


\section{Conclusions}

The proposed method enables the monitoring of plant survival with UAVs in afforestation projects employing water storage devices such as Waterboxx. This method provides the georeferencing of gaps or possible gaps for their replenishment and provides complete information on the reforested plot with greatly reduced field work. The UAV monitoring procedure can be particularly useful in hazardous areas that are difficult to access on foot. The flight path must be planned according to the particular conditions of the terrain to ensure transversal and longitudinal overlapping and to avoid possible balancing problems due to the wind. The combination of a conventional RGB camera with a modified camera to capture the NIR channel and the synchronized single-shot technology achieve greater reliability by precluding the need to conduct different flights with each camera, which would produce radiometric variations due to the time lag (i.e., due to differences in plant projections, solar height, clouds, etc.). A disadvantage of this method is that the weight of both cameras requires the use of a multi-rotor instead of a fixed-wing drone, limiting the performance of the method in larger study areas. Advances in technology will solve this problem as new camera models become increasingly lighter and provide higher resolution.

The application of indices based on plant vigour and chlorophyll levels (NDVI, G-NDVI and SAVI) obtained satisfactory results for well-developed plants or species with more foliage but did not allow such clear discrimination in small plants. The PCA-based "decorrelation stretch" algorithm, combined with Sobel filters, provided the best results to correctly identify the state of most seedlings. This PCA-based transformation can be very efficient for visual interpretation. 
The test results showed high overall accuracy $(96.2 \%)$ with a flight height of $109 \mathrm{~m}(\sim 2.74 \mathrm{~cm}$ pixel1 GSD), with various species of both conifers and hardwoods that never exceeded $60 \mathrm{~cm}$. However, the stage of development of the plant (height and leaf development) vs flight height must be considered. It is expected that flying at lower altitudes allows to identify the status of smaller seedlings, obtaining close accuracy rates if the GSD remains within an approximate range of 2.5-3 $\mathrm{cm}$.

The particular shape and the plastic material flap of the Waterboxx device, which is radiometrically uniform, facilitate the detection of vegetation, especially of larger plants. However, this design hampers the discrimination of small seedlings $(<10 \mathrm{~cm}$ tall $)$ because of the spectral response produced by the central aperture of the device. This problem diminishes over time as plants increase in size.

\section{Acknowledgements}

This work was supported by the Xunta de Galicia under the Grant "Financial aid for the consolidation and structure of competitive units of investigation in the universities of the University Galician System (2016-18)” [ED431B 2016/030, ED341D R2016/023] and the European Program Life+ [LIFE/ENV/ES/000447] “The Green Deserts: New planting techniques for tree cultivation in desertified environments to face Climate Change". 


\section{References}

Agapiou A, Hadjimitsis DG, Alexakis DD. 2012. Evaluation of broadband and narrowband vegetation indices for the identification of archaeological crop marks. Remote Sens. 4:3892-3919.

Agüera-Vega, F, Carvajal-Ramírez, F., Martínez-Carricondo, P. (2016) Accuracy of digital surface models and orthophotos derived from unmanned aerial vehicle photogrammetry. J of Survey Eng 143.2: 04016025.

Bainbridge DA. 2002. Alternative irrigation systems for arid land restoration. Ecol Restor. 20:23-30.

Del Campo A, Merce G, Ceacero CJ, Navarro R. 2013. Evaluación de depósitos de agua de liberación lenta (Waterboxx) en el establecimiento de repoblaciones en áreas adversas de la Comunidad Valenciana. In: 6th Spanish For Congr. Vitoria-Gasteiz; p. 1-11.

Chuvieco E, Martín MP, Palacios A. 2002. Assessment of different spectral indices in the red-near-infrared spectral domain for burned land discrimination. Int J Remote Sens. 23:5103-5110.

Cristiano P, Madanes N, Campanello P, Di Francescantonio D, Rodríguez S, Zhang YJ, Carrasco O, Goldstein G. 2014. High NDVI and Potential canopy photosynthesis of South American subtropical forests despite seasonal changes in leaf area index and air temperature. Forests. 5:287-308.

Eisenbeiß H. 2009. UAV photogrammetry.: ETH Zürich.

García-Valdés R, Svenning JC, Zavala M, Purves DW, Araújo MB. 2015. Evaluating the combined effects of climate and land-use change on tree species distributions. J Appl Ecol [Internet]. 52:902-912. Available from: http://doi.wiley.com/10.1111/1365-2664.12453

Gillespie AR, Kahle A, Walker R. 1986. Color enhancement of highly correlated images. I. Decorrelation and 
HSI contrast stretches. Remote Sens Environ. 20:209-235.

Gitelson AA, Merzlyak MN. 1997. Remote estimation of chlorophyll content in higher plant leaves. Int J Remote Sens [Internet]. 18:2691-2697. Available from: http://www.scopus.com/record/display.url?eid=2s2.0-0031205878\&origin=inward\&txGid=yhIy9MQ1WOT0E_0PAAqWCeR\%3A17

Gnädinger F, Schmidhalter U. 2017. Digital counts of maize plants by Unmanned Aerial Vehicles (UAVs). Remote Sens. 9.

Haase D, Rose R. 1993. Soil moisture stress induces transplant shock in stored and unstored 2+ 0 Douglas-fir seedlings of varying root volumes. For Sci. 39:275-294.

Helman D, Lensky I, Tessler N, Osem Y. 2015. A phenology-based method for monitoring woody and herbaceous vegetation in mediterranean forests from NDVI time series. Remote Sens. 7:12314-12335.

Huete AR. 1988. A soil-adjusted vegetation index (SAVI). Remote Sens Environ. 25:295-309.

Huete AR, Jackson RD. 1987. Suitability of spectral indices for evaluating vegetation characteristics on arid rangelands. Remote Sens Environ. 23.

Hunt ER, Cavigelli M, Daughtry C, McMurtrey J, Walthall C. 2005. Evaluation of digital photography from model aircraft for remote sensing of crop biomass and nitrogen status. Precis Agric. 6:359-378.

Johnson S, Mudrak E, Beever E, Sanders S, Waller D. 2008. Comparing power among three sampling methods for monitoring forest vegetation. Can J For Res Can Rech For. 38:143-156.

Lehmann J, Nieberding F, Prinz T, Knoth C. 2015. Analysis of unmanned aerial system-based CIR images in forestry-a new perspective to monitor pest infestation levels. Forests. 6:594-612.

Li X, Shao G. 2014. Object-based land-cover mapping with high resolution aerial photography at a county scale in midwestern USA. Remote Sens. 6:11372-11390. 
Liu M, Li Z, Ren W. 2014. Research on the Effect of Waterboxx Technology on Haloxylon Ammodendron Afforestation in Arid and Semiarid Areas. Adv Sci Eng Med. 6:236-239.

Lloret F, Lobo A, Estevan H, Maisongrande P, Vayreda J, Terradas J. 2007. Woody plant richness and NDVI response to drought events in Catalonian (northeastern Spain) forests. Ecology. 88:2270-2279.

Marcos-Robles J, Ortiz-Sanz L, Garrido-Laurnaga F, Clérigo-Pérez Z, Hernández-Navarro S, Gil-Docampo M, Ortiz-Sanz J. 2013. Efecto termoaislante del Waterboxx en las repoblaciones forestales. In: 6th Spanish For Congr.; p. 1-14.

Mesas-Carrascosa, FJ., Torres-Sánchez, J., Clavero-Rumbao, I., García-Ferrer, A., Peña, J. M., Borra-Serrano, I., \& López-Granados, F. (2015). Assessing optimal flight parameters for generating accurate multispectral orthomosaicks by UAV to support site-specific crop management. Remote Sens. 7.10: 12793-12814.

Perea-Moreno AJ, Aguilera-Ureña MJ, Meroño-De Larriva JE, Manzano-Agugliaro F. 2016. Assessment of the Potential of UAV Video Image Analysis for Planning Irrigation Needs of Golf Courses. Water. 8:584.

Salamí E, Barrado C, Pastor E. 2014. UAV Flight Experiments Applied to the Remote Sensing of. :1105111081.

Singh A, Singh S. 2011. Forest Vegetation Analysis and Land Cover Assessment in Tan Sub Watershed of Hasdeo River Basin, Chhattisgarh, India Forest Vegetation Analysis and Land Cover Assessment in Tan Sub Watershed of Hasdeo River Basin, Chhattisgarh, India. In: FIG Work Week 2011.; p. 18-22.

Sobel I, Feldman G. 1973. A 3x3 isotropic gradient operator for image processing. Hart, P E Duda R O Pattern Classif Scene Anal.:271-272.

Tang J, Folmer H, Xue J. 2015. Technical and allocative efficiency of irrigation water use in the Guanzhong Plain, China. Food Policy. 50:43-52. 
Tonkin, T., Midgley, N. (2016). Ground-Control Networks for Image Based Surface Reconstruction: An Investigation of Optimum Survey Designs Using UAV Derived Imagery and Structure-from-Motion Photogrammetry. Remote Sens, 8(786), 16-19.

Tucker CJ. 1979. Red and photographic infrared linear combinations for monitoring vegetation. Remote Sens Environ. 8:127-150.

Zhou G. 2014. Determination of green aboveground biomass in desert steppe using litter-soil-adjusted vegetation index. Eur J Remote Sens. 47:611-625. 
